\title{
Quiste dentígero. Reporte de un caso
}

\author{
Dentigerous cyst. A case report
}

\author{
Dra. Roxana G. Spini ${ }^{a}$ Dr. Lucas Bordino ${ }^{a}$, Dr. Daniel Cruz ${ }^{a}$, Dra. María de los Ángeles Fitz Maurice \\ Dra. Andrea Martins ${ }^{a}$ y Dr. Julián Michalski ${ }^{a}$
}

\begin{abstract}
RESUMEN
Los quistes del maxilar constituyen un conjunto muy variado de entidades, entre las que se incluyen los tumores benignos y malignos deorigen odontogénico. Existe poca información sobre la prevalencia de esta patología. Afecta con mayor frecuencia al sexo masculino, entre la segunda y la tercera década de la vida. La proporción de pacientes de 6 a 7 años con quistes dentígeros es de solo 9,1\%.

El quiste dentígero engloba la corona de un diente permanente normal, impactado, no erupcionado. Suele presentar una sintomatología insidiosa y no se descubre hasta que la tumoración afecta a los órganos vecinos.

El objetivo es exponer un caso de quiste dentígero. Si bien es de aparición muy poco frecuente, la presentación de este caso sirve para poner al médico pediatra en conocimiento sobre cómo debe actuar ante una tumoración maxilar unilateral en un niño sano. Se remarca la importancia del manejo multidisciplinario de la entidad.

Palabras clave: quiste odontogénico, quiste dentígero, maxilar, tumores odontogénicos, odontología pediátrica.
\end{abstract}

\begin{abstract}
Maxillary cysts are a diverse group of entities that include benign and malignant odontogenic tumors. Information on the prevalence of this disease is limited. It is more common among males, and usually occurs in the second and third decade of life. The proportion of 6 to 7 year old patients with dentigerous cysts is only $9.1 \%$.

Dentigerous cysts encompass the crown of a permanent and unerupted impacted teeth. They are usually slow growing asymptomatic lesions that are not discovered until they affect surrounding organs.

The aim of this study is to present an unusual case of dentigerous cyst and to inform the pediatrician about the management of a unilateral maxillary tumor in a healthy child, underlining the importance of a multidisciplinary approach of this disease. Key words: odontogenic cyst, dentigerous cyst, maxilla, odontogenic tumors, pediatric dentistry.
\end{abstract}

http:/ /dx.doi.org/10.5546/aap.2016.e338

a. División de Otorrinolaringología del Hospital General de Niños Pedro de Elizalde. Ciudad Autónoma de Buenos.

Correspondencia:

Dra. Roxana G. Spini: spiniroxana@intramed.net

Financiamiento: Ninguno.

Conflicto de intereses: Ninguno que declarar.

Recibido: $17-12-2015$

Aceptado: 2-3-2016

\section{INTRODUCCIÓN}

Los quistes maxilares (QM) constituyen un conjunto variado de entidades de origen odontogénico y no odontogénico, benignos y malignos. ${ }^{1}$ Si bien son poco habituales, su frecuencia es mayor en adultos. Los quistes odontogénicos representan, aproximadamente, el 35\% de los QM. ${ }^{1}$

El manejo debe ser multidisciplinario: pediatría, otorrinolaringología, cirugía maxilofacial y odontología. ${ }^{2}$

Ante una asimetría facial, debemos diferenciar los procesos originados a nivel maxilar, como masas óseas, lesiones fibroóseas y quistes, de las estructuras circundantes, como afecciones de la glándula parótida, contenido de la órbita y estructuras dentales. ${ }^{2}$

El objetivo del presente artículo es presentar las características clínicas y el manejo de un paciente con diagnóstico de quiste dentígero (QD) para poner al médico pediatra en conocimiento sobre cómo debe actuar ante la aparición de una tumoración maxilar unilateral en un niño sano. Se remarca la importancia del manejo multidisciplinario de la entidad.

\section{CASO CLÍNICO}

Paciente de 11 años de edad, masculino, previamente sano. Consultó a nuestro Servicio por tumoración maxilar izquierda de 2 meses de evolución. Se encontraba en buen estado general, eutrófico y afebril.

Al momento del examen físico, se observaba una asimetría facial en la región malar izquierda, de consistencia dura, bordes difíciles de delimitar, de, aproximadamente, $4 \times 5 \mathrm{~cm}$ de diámetro, $\sin$ signos de flogosis ni dolor. En la cavidad oral, presentaba abombamiento del paladar duro con predomino del lado izquierdo (Figura 1), mientras que, en la región gingivolabial superior izquierda, alternaban zonas duras y fluctuantes (Figura 2). Tenía buen estado dentario y adecuada oclusión. En la fosa nasal izquierda, se observaba un desplazamiento del piso y de la pared intersinusonasal, que ocluía parcialmente la luz. No se evidenció compromiso neurológico ni 
ocular. No presentaba adenopatías regionales. El resto del examen físico era normal.

Se realizó una radiografía mentonasoplaca y oclusal (Figura 2), donde se observaba velamiento del seno maxilar izquierdo y pieza dentaria incluida dentro del seno. El análisis de laboratorio (hemograma, eritrosedimentación y coagulograma) estaba dentro de los parámetros normales. El Servicio de Odontología revisó cada pieza dentaria y constató la falta del canino izquierdo.

En la tomografía, se evidenciaba una extensa lesión lítica que ocupaba el seno maxilar izquierdo y afectaba a la pared anterior, posterior y lateral, así como también al reborde alveolar homolateral. La pared interna del seno maxilar obstruía la región osteoinfundibular. Dentro de la tumoración, se identificaba una imagen de densidad similar a las piezas dentarias (Figura 3).

Con el diagnóstico presuntivo de quiste odontogénico, se decidió la cirugía junto con el cirujano maxilofacial. Por técnica de CaldwellLuc, se realizó la exéresis completa de la cápsula del quiste y dos piezas dentarias incluidas en él, aspirándose previamente contenido serosanguinolento (Figura 4). Se dejó un taponaje con gasa furacinada en la cavidad por 48 horas. El informe de anatomía patológica fue quiste odontogénico dentígero.

El seguimiento continúa hasta el día de la fecha semestralmente, sin recidiva luego de un año de su diagnóstico.

\section{DISCUSIÓN}

Los QM representan una de las principales causas de destrucción de los maxilares. ${ }^{3}$ Según la Organización Mundial de la Salud (OMS), los QM se clasifican en dos grandes grupos: neoplasias y quistes, que pueden ser epiteliales y no epiteliales. Los quistes epiteliales pueden ser subdivididos en odontogénicos (Tabla 1) 4,5 y no odontogénicos. El QD se ubica dentro de los quistes epiteliales odontogénicos.

FIGURA 2. Radiografía frontonasoplaca: senomaxilarizquierdo velado con una imagen dentaria en su interior (flecha).

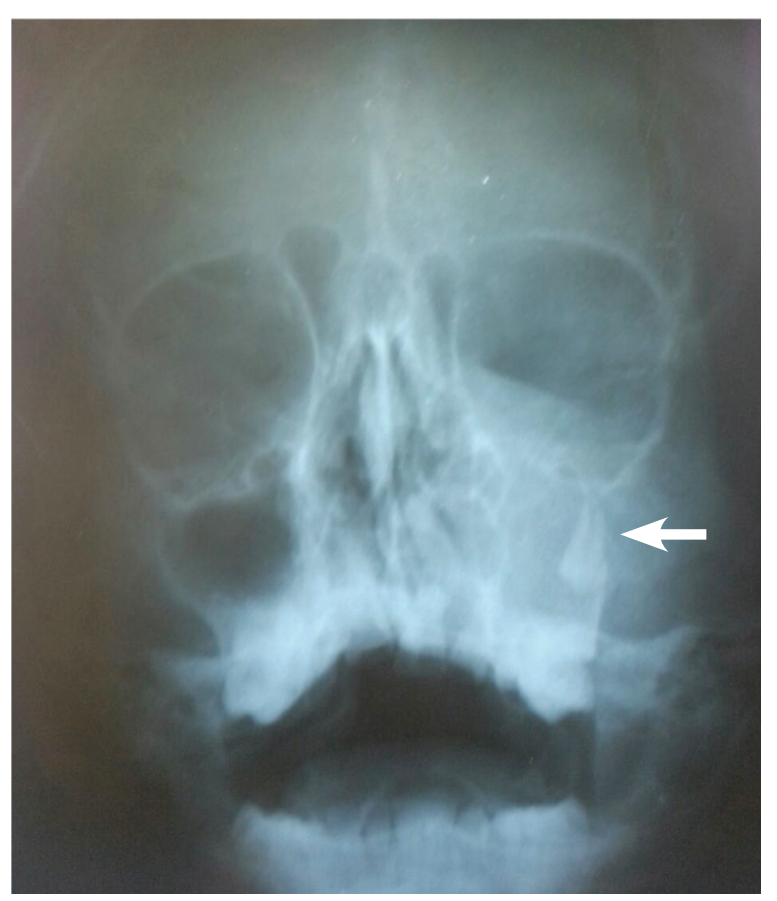

Figura 1. A. Asimetría facial. Tumoración del maxilar izquierdo. B. Se visualiza un abombamiento del paladar duro homolateral a la tumoración. C. Región gingivoyugal superior izquierda: tumoración con zonas duras y fluctuantes.
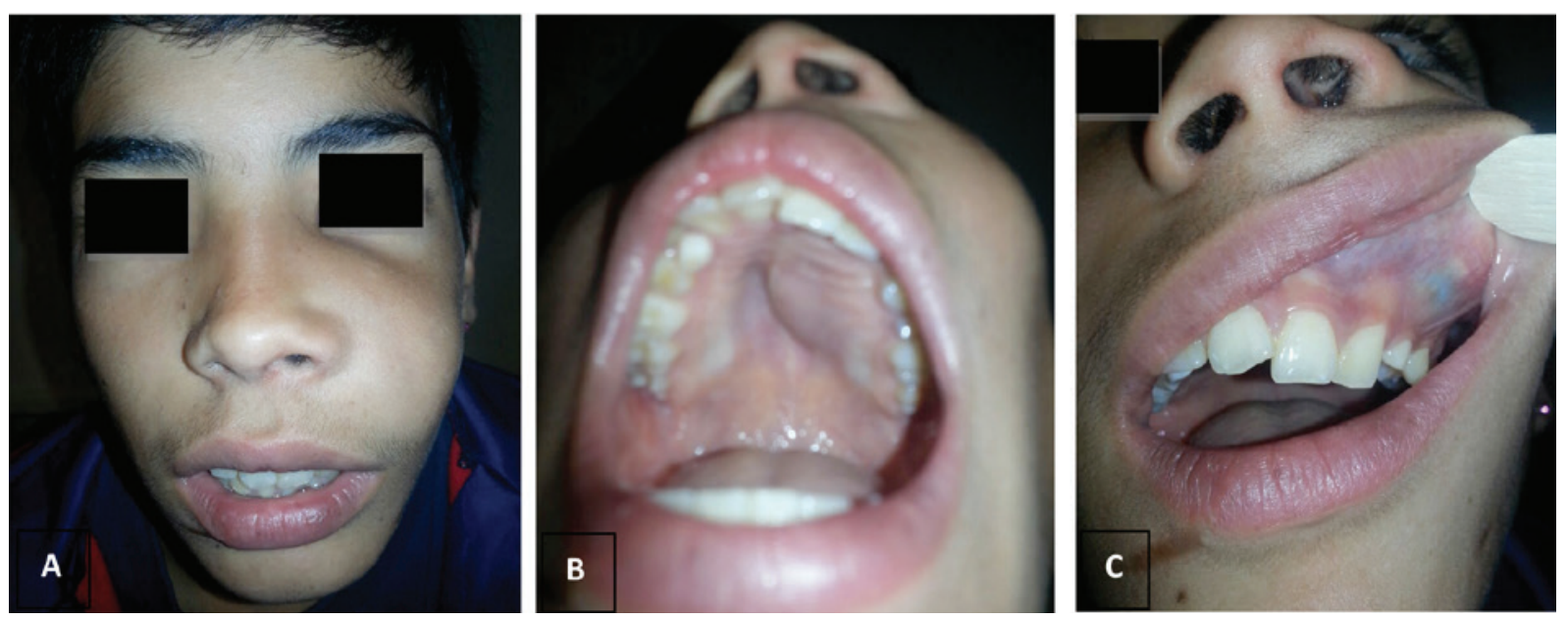
Existe poca información acerca de la prevalencia de los QM. En una revisión de 40000 biopsias, Daley y sus cols., encontraron 6879 quistes odontogénicos $(17,2 \%)$, de los cuales $4468(64,9 \%)$ correspondieron a quistes radiculares; $1662(24,1 \%)$, a QD; y solamente 335 $(4,8 \%)$, a queratoquistes. El resto de las entidades reconocidas por la OMS se observaron en menos del $2 \%$ cada una. ${ }^{6}$

FIgURA 3. Tomografía computada: lesión lítica que ocupa el seno maxilar izquierdo y compromete sus paredes y la región alveolar homolateral. A. Corte axial. La flecha señala la pieza dentaria. B. Corte coronal. La flecha señala el compromiso de la pared interna que obstruye la región osteoinfundibular.
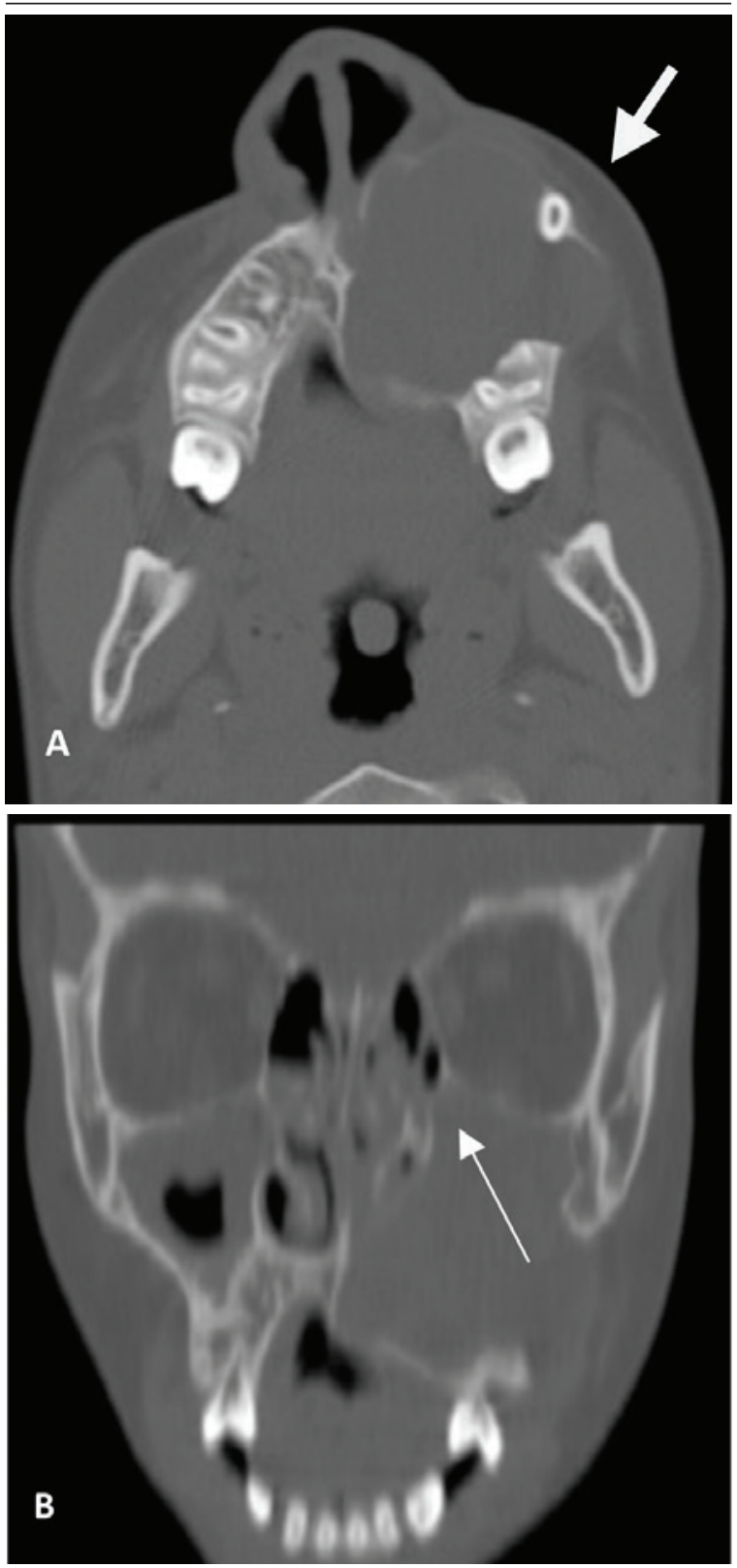

Los QM son lesiones óseas destructivas de origen inflamatorio o residuos embrionarios secundarios a una alteración en el desarrollo (epiteliales, mesenquimales o mixtos), de ocurrencia común en los senos maxilares. Sus variantes más usuales son el quiste radicular, el QD y el queratoquiste odontogénico. ${ }^{7}$

Los quistes radiculares son lesiones de origen inflamatorio que se encuentran asociadas a dientes desvitalizados por las secuelas de caries. ${ }^{3}$

El QD engloba la corona de un diente y se une a su cuello. ${ }^{1}$ Afecta con mayor frecuencia al sexo masculino en una relación 2:1, entre la segunda y la tercera década de la vida. La proporción de pacientes de 6 a 7 años con QD es de solo 9,1\%. Se encuentra asociado a la corona de un diente permanente normal, impactado, no erupcionado; rara vez, se presenta en dientes primarios. Se localiza, con mayor frecuencia, a nivel del tercer molar inferior, seguido del canino superior $\mathrm{y}$, por último, del segundo premolar superior. . $^{1,57-9}$

TABla 1. Quistes odontogénicos

\begin{tabular}{lc}
\hline Lesiones del desarrollo & Quistes de origen inflamatorio \\
\hline $\begin{array}{ll}\text { 1. Quiste gingival del } \\
\text { recién nacido }\end{array}$ & 1. Quiste radicular \\
$\begin{array}{ll}\text { 2. Queratoquiste odontogénico } & \text { 2. Quiste residual } \\
\text { 3. Quiste dentígero } & \begin{array}{l}\text { 3. Quiste paradental y bucal } \\
\text { mandibular infectado }\end{array} \\
\text { 4. Quiste de erupción } & \\
\text { 5. Quiste lateral periodontal } & \\
\text { 6. Quiste gingival del adulto } & \\
\text { 7. Quiste odontogénico glandular }\end{array}$ \\
\hline
\end{tabular}

Figura 4. Técnica de Caldwell-Luc: exéresis del diente retenido con su cápsula.

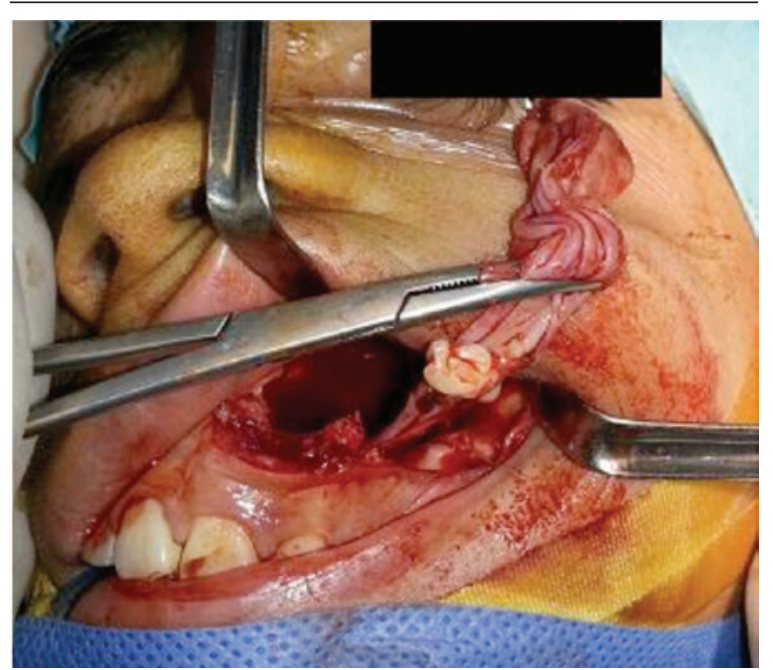


Existen varias teorías que tratan de explicar el origen del QD:

- Acumulación de líquido una vez que la corona del diente se ha formado por completo.

- Origen extrafolicular del QD, que comienzan como quistes periapicales en dientes primarios y luego crecen y engloban el germen del diente permanente.

- Origen durante su odontogénesis.7

Los QM tienen una sintomatología clínica común. Suelen ser de presentación insidiosa y no se descubren hasta que afectan a los órganos vecinos. En el 95\% de los casos, el síntoma principal es la tumefacción facial o palatina, seguida por desplazamientos y dolores dentales. En caso de ser palpables, suelen presentar una cápsula depresible (signo de Dupuytren). Cuando afectan al seno maxilar, pueden manifestarse por dolores infraorbitarios, sinusitis maxilar unilateral crónica u obstrucción nasal unilateral. Todas estas lesiones se pueden complicar cuando se extienden fuera del seno maxilar y afectan al seno etmoidal, la base del cráneo, la fosa pterigopalatina y/o la fosa infratemporal. ${ }^{1,5,10}$

Los QD se caracterizan por el agrandamiento maxilar y una imagen quística con el diente no erupcionado y el desplazamiento de los dientes vecinos. ${ }^{1}$ Pueden alcanzar grandes dimensiones y destruir las raíces de los dientes adyacentes. Su pared está formada por tejido conjuntivo y por un epitelio. . $^{1,7}$,

Radiológicamente, representan una imagen radiolúcida uni- o multilocular, bien circunscrita, que rodea la corona de un diente incluido, por lo general, desplazado. La presencia de una imagen mayor de $2 \mathrm{~mm}$ es sugestiva de QD. ${ }^{7,8}$

Es indispensable realizar una tomografía computada antes de adoptar cualquier decisión terapéutica, ya que permite apreciar su ubicación exacta, volumen y relaciones con los tejidos adyacentes, así como el estado de las corticales óseas. ${ }^{1,10}$ Debe solicitarse una resonancia magnética cuando la lesión se extienda a etmoides, fosa infratemporal. ${ }^{1}$

E 1 diagnóstico definitivo es anatomopatológico. Cuando múltiples QD son diagnosticados en un paciente, deben sospecharse síndromes genéticos. ${ }^{9,10} \mathrm{El}$ síndrome de GorlinGoltz presenta una tríada clásica: múltiples carcinomas basocelulares, quistes mandibulares y anomalías esqueléticas. La displasia cleidocraneal tiene un fenotipo característico con cráneo grande y braquiocefálico, hipoplasia o aplasia clavicular y anomalías dentales, que consisten en el retraso en la erupción de los dientes y la presencia de múltiples piezas supernumerarias que permanecen impactadas de forma permanente en los maxilares. Por último, el síndrome de Gardner se caracteriza por la asociación de pólipos colorrectales, quistes epidérmicos cutáneos y osteomas mandibulares y de huesos largos. ${ }^{11-13}$

El tratamiento consiste en la extirpación del quiste con los dientes flotantes y el germen dentario, según la necesidad, que proporciona la curación en todos los casos. Después de la enucleación completa, es rara su recidiva. Excepcionalmente, puede desarrollarse en su pared un ameloblastoma o un carcinoma. ${ }^{1}$

Es importante destacar la interconsulta entre las diferentes especialidades y odontología, cuando evalúa a niños y adolescentes que se encuentran en el período de recambio dentario, momento en que es posible la presentación de lesiones patológicas. ${ }^{7}$ Asimismo, se debe controlar el estado bucal, ya que las caries no tratadas pueden producir inflamación y generar, por ejemplo, el quiste radicular, que es el primero en frecuencia.

En una revisión de 800 casos diagnosticados como quistes odontogénicos, el 12\% tenían entre 0 y 14 años y el $72 \%$ eran varones, con una media de 9,78 años. El quiste de mayor incidencia fue el QD (57\%), seguido del quiste radicular (31\%). Se manifestaron como tumoración maxilar indolora en la mayoría de los casos; solo el $10 \%$ eran dolorosos. $^{10}$

La presentación clínica de nuestro paciente coincide con la mayoría de las manifestaciones publicadas en la bibliografía mundial.

Para finalizar, queremos resaltar la importancia en la derivación oportuna al odontólogo y otorrinolaringólogo ante una asimetría facial. Si bien la mayoría son lesiones benignas, pueden generar gran morbilidad por destrucción y deformidad facial a largo plazo.

\section{REFERENCIAS}

1. Hervé S, Conessa C, Chollet O, Poncet JL. Quistes del maxilar. EMC - Otorrinolaringología 2004;33(1):1-8.

2. Molina $\mathrm{O}$, Rodríguez Azrak MS. Tumor odontogénico en seno maxilar. Rev Hosp Niños B Aires 2011;53(242):167-71.

3. Mosqueda Taylor A, Irigoyen Camacho M, Díaz Franco M, Torres Tejero M. Quistes odontogénicos. Análisis de 856 casos. Med Oral 2002;7(2):89-96.

4. Kramer IR, Pindborg JJ, Shear M. Histological typing of odontogenic tumours. Berlin: Springer-Verlag; 1992.

5. De Vicente Rodríguez JC, López-Arranz J. Quistes de los maxilares. In Suárez Nieto C. Tratadode Otorrinoloringologíay cirugía de cabeza y cuello. 2. ${ }^{\mathrm{a}}$ ed. Buenos Aires: Panamericana; 2008.Págs.3689-706. 
6. Daley TD, Wysocki GP, Pringle GA. Relative incidence of odontogenic tumors and oral and jaw cysts in a Canadian population. Oral Surg Oral Med Oral Pathol 1994;77(3):276-80.

7. Caballero HA. Quistes y tumores del maxilar. Rev Cir Infantil 1999;9(4):205-10.

8. Romero Y, Jiménez C,HernándezP, Alexis G. Presentación inusual de un quiste dentigero en paciente pediátrico. Reporte de un caso y revisión de la literatura. Acta Odontol Venez 2007:45(2):1-8.

9. Quintana DíazJC,AliZwiad A,LópezLazoS, Vega BasultoS, etal. Quiste dentígeno gigante en una niña. Presentación de un caso. Rev Cubana Estomatol [Online] 2007;44(2). [Acceso: 23 de enero de 2016]. Disponible en: http: / /scielo.sld.cu/ scielo.php?pid=S0034-75072007000200008\&script $=$ sci arttext.
10. Pina Godoy G, Silveira EJD, Gordón-Nuñez M, Guedes Queiroz L, Dantas Gomes D. Quistes de los maxilares en niños: un análisis clínico. Acta Odontol Venez 2007: 45(4): $1-7$.

11. Rosón-Gómez S, González-García R, Naval-Gías L, SastrePérez J, et al. Síndrome de Gorlin-Goltz: Serie de 7 casos. Rev Esp Cir Oral Maxilofac 2009;31(5):309-15.

12. Olmos Martínez J, Martínez García J, González Macías J. Displasia Cleidocraneal. Rev Esp Enfer Metab Oseas 2007;16(4):82-3.

13. Chimenos-Küstner E, Pascual M, Blanco I, Finestres F. Poliposis familiar hereditaria y síndrome de Gardner: Aportación de la exploración odontoestomatológica a su diagnóstico y descripción de un caso. Med Oral Patol Oral Cir Bucal 2005;10(5):402-9. 